\title{
The Potential Effect of the Angiotensin-Converting Enzyme 2 (ACE2) Receptor of 2019-nCoV in Lung Adenocarcinoma Patients on Age
}

Qin Huo

Shenzhen Second People's Hospital

Zhenwei Li

Shenzhen Second People's Hospital

Ling Shao

Shenzhen Second People's Hospital

Siqi Chen

Guangzhou Medical University

Jiaying Li

University of south china

Ni Xie ( $\nabla$ xn100@szu.edu.cn )

Shenzhen Second People's Hospital https://orcid.org/0000-0002-7167-9343

Primary research

Keywords: 2019-nCoV, ACE2, expression, lung adenocarcinoma, clinical-pathological parameters

Posted Date: September 22nd, 2020

DOl: https://doi.org/10.21203/rs.3.rs-78749/v1

License: (9) (i) This work is licensed under a Creative Commons Attribution 4.0 International License.

Read Full License 


\section{Abstract}

\section{Background}

The 2019-nCoV epidemic is the public health emergency that has had the greatest impact on the world. Our study aimed to better understand the underlying mechanisms and function of angiotensin-converting enzyme 2 (ACE2) receptor of 2019-nCoV on lung adenocarcinoma patients (LUAD), and provide a theoretical basis for early diagnosis, prognosis and targeted therapy of 2019-nCoV.

\section{Methods}

This study focuses on the expression level, functions, mutation rate, and copy number variations (CNVs) of ACE2 in LUAD using an extensive bioinformatics data mining process. The interaction between ACE2 expression and clinical-pathological parameters of patients with LUAD was investigated using UALCAN. Also, the essential biological features, single nucleotide variations (SNVs), CNVs, and pathway activities of genes interacting with ACE2 in these cancers were further analyzed.

Results

We found that ACE2 expression in LUAD patients increased with age, but it was not related to cancer status, patient's race, patient's gender, or patient's smoking habits. Moreover, our results showed that compared to that in normal tissues, ACE2 was highly expressed in colon adenocarcinoma (COAD), kidney renal papillary cell carcinoma (KIRP), pancreatic adenocarcinoma (PAAD), rectum adenocarcinoma (READ), and stomach adenocarcinoma (STAD). However, there is no significant difference in the expression of ACE2 in patients of different ages.

\section{Conclusions}

These findings demonstrate the importance of ACE2 in LUAD, and provide insights into the regulatory mechanisms and function of ACE2.

\section{Background}

During the Spring Festival of 2020, the outbreak of pneumococcal infection caused by the 2019 coronavirus disease (2019-nCoV) spread rapidly in China and many countries. Subsequently, the virus was found to spread through interpersonal communication was found when a group of 2019-nCov patients with a Wuhan travel record of patients appeared to travel more cities in China [1-3]. On January 21, 2020, The World Health Organization (WHO) announced that 2019-nCoV could be maintained through interpersonal communication [WHO. https://www.who.int/emergencies/diseases/novel-coronavirus2019.]. By July 19 , the National Health Commission had received reports of 83,682 confirmed cases and 4,634 deaths in 31 provincial-level regions and the Xinjiang Production and Construction Corps on the Chinese mainland, and in all 78,799 patients had been cured and discharged from hospital (http://en.nhc.gov.cn/2020-03/11/___77599.htm). The epidemic spreading all over China has brought 
strong shock. In the face of the new coronavirus epidemic, the whole country has made concerted efforts to treat patients and prevent its spread. With the accumulation and analysis of more cases, people's understanding of 2019-nCoV is gradually deepening. To date, there is still no specific medicine for 2019$\mathrm{nCoV}$, and it is urgent to conduct in-depth research on its pathogenic mechanisms to promote the development of effective control measures. To prevent further spread of the epidemic, a nationwide campaign was launched.

2019-nCoV infection of the human body requires binding to receptors expressed by host cells, and although the virus can invade the human body in many different ways, invading the lungs through the respiratory tract and causing severe pneumonia is still the main mode. Previous studies demonstrated that angiotensin-converting enzyme 2 (ACE2) is a receptor for SARS coronavirus (SARS-CoV) and the novel coronavirus 2019-nCoV/SARS-CoV-2 [4, 5]. As reported, ACE2 is expressed in the lung, heart, kidney, and intestine [6]. Zhang $\mathrm{H}$ et al. revealed that ACE2 was not only the entry receptor of the virus, but also had a protective effect on lung injury [7]. Moreover, Hofmann, $\mathrm{H}$. et al. revealed a positive correlation between ACE2 expression and SARS-CoV infection in vitro [8]. Recent work has indicated that Asian males may have higher expression levels of ACE2 [9]. However, Chen, Y. et al. found that there was no significant difference in the expression of ACE2 in Asians compared with other races, but the expression was positively correlated with age [10]. Whether the expression of ACE2 is higher in patients with lung adenocarcinoma (LUAD) remains to be studied. Therefore, more attention should be paid to the expression of ACE2 in patients with LUAD. In addition, the relationship between ACE2 expression and clinical and pathological parameters, such as individual cancer status, patient's race, patient's gender, patient's age, patient's smoking habits, node metastasis status, and histological subtypes, is unclear. It is necessary to perform a more accurate analysis of the expression of ACE2, which was the aim of the current study.

This study focused on the correlation between ACE2 expression and clinicopathological parameters, and we described the functions, mutation rates, copy number variation (CNVs), and genomic alterations of ACE2 in LUAD. We next statistically analyzed the proteins interacting with ACE2 and Gene Ontology (GO) enrichment and Kyoto Encyclopedia of Genes and Genomes (KEGG) pathway analysis. Moreover, we investigated ACE2 expression in other cancers, including colon adenocarcinoma (COAD), kidney renal papillary cell carcinoma (KIRP), pancreatic adenocarcinoma (PAAD), rectum adenocarcinoma (READ), and stomach adenocarcinoma (STAD). We then discussed the essential biological features, single nucleotide variations (SNVs), CNVs, and pathway activities of the genes interacting with ACE2 in these cancers. The findings of this study will help enhance the understanding of the potentially positive role of ACE2 in LUAD and provide a theoretical basis for the early diagnosis, prognosis, and targeted therapy of 2019-nCoV.

\section{Methods}

\section{Analysis of the mutation rate and CNVs distribution of ACE2 analysis in LUAD}


DriverDBv3 (http://driverdb.tms.cmu.edu.tw/) is a cancer database that incorporates somatic mutation, methylation, copy number variation, and clinical data in addition to annotation bases. This database can help researchers visualize the relationships between cancers and driver genes [11]. The mutation squares indicate the number of mutation tools that identify this gene as a mutation driver. As the number of tools goes from low to high, the blue color goes from light to deep. The CNVs squares indicate the CNVs gain or loss of a gene. Red represents gain (1) and the green represents a loss (-1). In this study, we used the DriverDBv3 tool to determine the mutation rate and CNVs distribution of ACE2 and their correlations with LUAD.

\section{Analysis of the protein network of ACE2}

To better understand the function of related proteins and understand their regulatory mechanisms more clearly. We predicted the protein-protein interactions of ACE2 vie the STRING database (https://string$\mathrm{db} .0 \mathrm{~g} /$ ), which is a system that searches for known and predicted protein-protein interactions [12]. The interactions include both direct physical interactions between proteins and indirect functional correlations between proteins. Besides, we analyzed the $\mathrm{GO}$ enrichment (biological process, molecular function, and cellular component) and KEGG pathways of the genes interacting with ACE2 with the Enrichr online database [13].

\section{ACE2 expression and clinical-pathological parameter analysis in LUAD}

We investigated the interaction between ACE2 expression and the clinicopathological parameters of patients with LUAD using UALCAN (http://ualcan.path.uab.edu/index.html), which is a comprehensive, user-friendly, and interactive web resource for analyzing gene expression data using The Cancer Genome Atlas (TCGA) level 3 RNA-seq data and clinical data from 31 cancer types [14]. It provides gene expression and clinicopathological parameter information. In the study, we entered the target gene ACE2 on the homepage of the website, selected LUAD, and obtained the differential expression of ACE2 in pathological parameters (individual cancer status, patient's race, patient's gender, patient's age, patient's smoking habits, node metastasis status, and histological subtypes).

\section{Immunohistochemical staining}

This study was performed on archived tissues from 20 diagnosed cases of lung adenocarcinoma patients who underwent surgery in Shenzhen Second People's Hospital. All the patients signed the informed consent form. This study was approved by the Ethics Committee of Shenzhen Second People's Hospital in accordance with the principles of the Declaration of Helsinki. The tissue samples were fixed in 4\% paraformaldehyde and embedded in paraffin. Anti-ACE2 (1: 200, Affinity Biosciences) were used as primary antibodies. MXB was used to detect secondary antibodies. The expression density of TACC3 in lung adenocarcinoma tissue was quantitated by scoring staining intensity, including negative $(-)$ and weak (+) staining, moderate (++) and strong (+++) staining, respectively $[15,16]$.

\section{ACE2 expression in other cancers}


We analyzed the expression of ACE2 in other cancers, including COAD, KIRP, PAAD, READ, and STAD compared to normal tissues using Gene Expression Profiling Interactive Analysis (GEPIA) database (http://gepia.cancer-pku.cn/), an interactive web application based on the gene expression analysis of 9,736 tumors and 8,587 healthy tissue samples from the TCGA and Genotype-Tissue Expression (GTEx) databases [17]. The correlation between ACE2 expression and patient's age was further analyzed using the UALCAN database.

\section{Gene Set Cancer analysis in LUAD}

GSCALite (http://bioinfo.life.hust.edu.cn/web/GSCALite/) is a very useful and important platform for gene set analysis in cancer [18]. In this GSCALite, we integrated the cancer genomics data from TCGA. The alterations in the DNA or RNA of cancer-related genes may contribute to cancer initiation, progression, diagnosis, prognosis, and therapy. We analyzed the gene set for SNVs: statistics, distribution, and types; CNVs: statistics of deletion/amplification of hetero/homozygous CNVs; and cancer pathway activity: the activity of 10 cancer-related pathways.

\section{Results}

\section{Genomic alterations of ACE2 in LUAD}

We then used the DriverDBv3 tool to determine the mutation rate and CNVs distribution of ACE2 and their correlations with LUAD. Data on the distribution of variants may help to further study the role of ACE2 in acute lung injury and lung function [19]. Fig. 1 A shows the mutation rate of ACE2 and its protein positions in LUAD. We found that the ACE2 protein has the highest mutation rate at positions 201-242. In contrast, the mutation rate of the ACE2 protein at 483-523 was the lowest. Additionally, a moderate mutation frequency occurs at the protein sites of 0-40, 80-121, 282-322, 644-684, and 765-805. The CNVs squares indicate the CNVs gain or loss of ACE2 in LUAD (Fig. 1B). We found the CNVs distribution mainly included gain, loss, none, and normal, and was positively correlated with ACE2 expression in LUAD (cor $=0.149, p=0.00075$ ). Among them, ACE2 expression was higher in copy number loss than in copy number gain.

\section{ACE2 protein network analyses}

Data from STRING were applied to determine the proteins interacting with ACE2 and the results are shown in Fig. 2. The following ten proteins were found to interact with ACE2: Angiotensinogen (AGT), Renin (REN), Neprilysin (MME), Dipeptidyl peptidase 4 (DPP4), Lysosomal Pro-X carboxypeptidase (PRCP), Meprin A subunit alpha (MEP1A), Type-1 angiotensin II receptor (AGTR1), Meprin A subunit beta (MEP1B), Xaa-Pro aminopeptidase 2 (XPNPEP2), and Type-2 angiotensin II receptor (AGTR2), and their correlation scores were $0.991,0.950,0.950,0.942,0.924,0.915,0.904,0.880,0.876$, and 0.858 , respectively.

\section{The enrichment analyses of ACE2}


To further explore the regulators of ACE2 in LUAD, we next statistically analyzed the significant GO enrichment terms and KEGG pathway of the identified genes via the Enrichr online database (Fig. 3, Table S2). The biological processes of these proteins were mainly involved in the regulation of systemic arterial blood pressure by renin-angiotensin (GO: 0003081), angiotensin maturation (GO: 0002003), and regulation of angiotensin levels in the blood (GO: 0002002). Regarding molecular functions, these proteins were mainly involved in the dipeptidyl-peptidase activity (GO: 0008239), aminopeptidase activity (GO: 0004177), and exopeptidase activity (GO: 0008238). The cell component analysis of these proteins showed that they were significantly enriched in invadopodium (GO: 0071437), azurophil granule membrane (GO: 0035577), and ficolin-1-rich granule membrane (GO: 0101003). Moreover, KEGG pathway analysis showed enrichment in the renin-angiotensin system, protein digestion and absorption, and renin secretion.

\section{Relationships between ACE2 expression and clinical-pathological parameters of patients with LUAD}

The goal of our study was to gain insights into the interaction between ACE2 expression and the clinicalpathological parameters of patients with LUAD (Table S1). To accomplish this, we first investigated ACE2 expression based on sample types, As shown in Fig. 4 A, the expression of ACE2 in primary samples was significantly higher than that in normal tissues $(p=2.16 \mathrm{E}-8)$. An analysis of individual cancer status showed that stage 1 , stage 2 , and stage 3 cancer tissues had significantly higher expression than that in normal tissues (normal-vs-stage 1: $p=1.81 \mathrm{E}-10$; normal-vs-stage 2: $\mathrm{p}=1.26 \mathrm{E}-03$; normal-vs-stage 3 : $\mathrm{p}=$ $2.48 \mathrm{E}-03)$, However, there was no significant difference between stage 4 and normal tissues $(p>0.05)$ (Fig. 4 B).

In comparing the patient's race (Fig. 4 C), we found that Caucasians and Asians with cancer had significantly higher ACE2 expression than normal control individuals (normal-vs-Caucasian: $p=1.55 \mathrm{E}-05$; normal-vs-Asian: $p=4.57 E-02)$. However, there was no significant difference in the expression of ACE2 among Caucasians, Asians, and African Americans ( $p>0.05)$.

In addition, we analyzed the relationship between ACE2 expression and patient's age (Fig. 4 D). Notably, we found that the expression of ACE2 in patients aged 61-80 years was significantly higher than that in patients aged 21-40 years $(p=7.23 E-04)$, the expression of ACE2 in patients aged 81-100 years was significantly higher than that in patients aged $21-40$ years $(p=3.68 \mathrm{E}-02)$, and the expression of ACE2 in patients aged 61-80 years was significantly higher than that in patients aged $41-60$ years $(p=1.60 \mathrm{E}-03)$. The results help explain why older people are more susceptible to SARS-CoV2.

Next, we investigated whether there was a difference between the expression of ACE2 and the patients' gender. As shown in Fig. 4 E, ACE2 expression was higher in both male and female cancer patients than that in the normal group (normal-vs-male: $p=6.17 \mathrm{E}-04$; normal-vs-female: $p=1.45 \mathrm{E}-09$ ), but no significant difference was found between ACE2 expression and sexes (male-vs-female: $p>0.05$ ).

Smoking is the most important risk factor for lung cancer [20]. The relationship between the expression of ACE2 and smoking in LUAD remains to be studied. Here, we focused on ACE2 expression according to 
patient's smoking habits (Fig. 4 F), including non-smoker, smoker, reformed smoker 1 ( $<15$ years), and reformed smoker 1 (> 15 years). Regarding the smoking habits of LUAD patients, the expression levels of ACE2 in patients with all conditions were higher than those in normal controls, but we found that there were no significant differences between patients' smoking habits and the expression of ACE2 $(p>0.01)$. Therefore, we speculate that the expression of ACE2 may not be related to the smoking habits of patients.

Subsequently, it is worth noting the relationship between ACE2 expression and node metastasis status (NO: no regional lymph node metastasis; N1: metastases in 1 to 3 axillary lymph nodes; N2: metastases in 4 to 9 axillary lymph nodes; and N3: metastases in 10 or more axillary lymph nodes). As shown in Fig. $4 \mathrm{G}$, we found that there was no significant difference between ACE2 expression and node metastasis status $(p>0.05)$.

Based on histological subtypes, the data showed that ACE2 expression was highest in lung clear cell adenocarcinoma (Clear Cell), but there was no significant difference compared with the normal group ( $p>$ 0.05). We found high ACE2 expression in the lung adenocarcinoma-not otherwise specified (NOS) and lung adenocarcinoma mixed subtype compared to normal controls in this cancer, with greater statistically significant (normal-vs-NOS: $p=4.38 \mathrm{E}-10$; normal-vs-mixed: $p=2.46 \mathrm{E}-03$ ) (Fig. $4 \mathrm{H}$ ). We also found that the expression of ACE2 was higher in lung mucinous adenocarcinoma compared to NOS in LUAD tumors $(p=7.67 \mathrm{E}-09)$.

Taken together, ACE2 expression in the lung increased with age, we further analyzed the expression of ACE2 in patient's age by immunohistochemistry were shown in Fig. 5. The expression level of ACE2 was quantified by scoring the intensity of staining, including negative $(-)$ and weak (+) staining, moderate (++) and strong (+++) staining. We found that ACE2 mainly localized in the plasma membrane and cytoplasm. ACE2 showed moderate expression in patients aged 81-100 years samples, and patients aged 61-80 years samples showed weak expression. However, the expression of ACE2 in patients aged 21-40 years and $41-60$ years was negative.

\section{ACE2 expression in other cancers}

To study the expression of ACE2 in other cancers and whether it is related to the patient's age. We examined the difference in ACE2 expression between tumor and adjacent normal tissues by using GEPIA (Fig. 6 A). We found that ACE2 was also highly expressed in COAD, KIRP, PAAD, READ, and STAD compared to normal tissues. These results suggested that the transcription level of ACE2 was cancer type-specific $(p<0.05)$. However, there was no significant difference in the expression of ACE2 among patients aged 21-40 years, 41-60 years, 61-80 years, and 81-100 years (Fig. 6 B).

\section{SNVs, CNVs, and pathway activity of hub proteins in LUAD}

To further understand the SNVs, CNV, and pathway activity of these proteins, we performed the analysis with GSCALite (Fig. 7 A-C). The SNVs module presented the SNVs frequency and variant types of these genes in LUAD. We found that the SNVs frequencies of MME, XPNPEP2, DPP4, AGTR1, and ACE2 were in 
the top five, and are $19 \%, 16 \%, 14 \%, 12 \%$, and $11 \%$, respectively. Among them, the variant types of ACE2 were missense mutations. In the CNVs module, the main copy number variants of ACE2 include heterozygous amplification and heterozygous deletion.

We then determined the pathway activity of these genes (Fig. 7 D-F). The pathways involved are apoptosis, cell cycle, DNA damage response, EMT, hormone AR, hormone ER, PI3K/AKT, RAS/MAPK, RTK (receptor tyrosine kinase), and TSC/mTOR. The results showed that RTK was activated by DPP4 and ACE2. The EMT pathway was mainly activated by AGTR1, DPP4, MME, and XPNPEP2. However, the cell cycle was mainly inhibited by AGTR1, DPP4, and PRCP. Besides, we found that the Hormone AR pathway was mainly inhibited by DPP4, AGTR1, XPNPEP2, MME, MEP1A, and ACE2.

\section{Discussion}

At present, the 2019-nCoV epidemic is the public health emergency that has had the greatest impact on the world and has received great attention from the international community. Facing the "encounter" of the epidemic, China responded positively, acted quickly, and took effective measures to resolutely curb the spread of the epidemic, which was highly appraised by the international community. Given that 2019nCoV pneumonia has become a new infectious disease transmitted from person to person, while working hard to comply with national instructions, we must work harder to understand the 2019-nCoV virus, and we should have a deeper understanding of how viruses invade the human body. A recent study showed that the ACE2 protein had a strong binding affinity with the spike protein of SARS-CoV-2 [21]. However, whether the expression of ACE2 is higher in patients with LUAD and whether it is related to the clinical and pathological parameters of patients is yet to be confirmed. In this study, we described the correlation between ACE2 expression and pathological parameters in LUAD and determined the proteins interacting with ACE2. Next, we analyzed SNVs, CNVs, and pathway activities of the hub genes in other cancers.

We statistically analyzed the proteins interacting with ACE2 and performed GO enrichment and KEGG pathway analysis. Among the ACE2 binging proteins, we found that AGT, REN, and MME had the highest correlation with ACE2 by STRING. Previous studies have suggested that AGT is abnormally methylated in gastric cancer and is associated with prognosis [22]. AGT was shown to inhibit vascular cell growth and angiogenesis [23]. MME, a zinc-metalloendopeptidase, has important roles in the physiology and pathology of many diseases such as cancer [24]. The biological processes of these proteins were mainly involved in the regulation of systemic arterial blood pressure by renin-angiotensin, angiotensin maturation, and regulation of angiotensin levels in the blood. Moreover, these proteins in this network are involved in different pathways, and KEGG pathway analysis showed enrichment in the renin-angiotensin system, protein digestion and absorption, and renin secretion. These data analysis results show that ACE2 is related to the regulation of systemic arterial blood pressure, providing a direction for further research.

Based on sample types, we found that the expression of ACE2 in primary samples was significantly higher than that in normal tissues. Notably, there was no significant difference between ACE2 expression 
and patient's smoking habits. Therefore, we speculate that the expression of ACE2 may not be related to the smoking habits of patients. In addition, our data showed that ACE2 expression was not related to the cancer stage or the patient's gender, node metastasis status, or histological subtypes. However, ACE2 expression in the lung increased with age, but there was no significant difference in the expression of ACE2 among Caucasians, Asians, and African Americans. This finding is consistent with the report from [10]. Furthermore, the representative immunohistochemical staining patterns for ACE2 were further verification, and high ACE2 expression was found in patients with advanced LUAD. The results help explain why older people are more susceptible to 2019-nCoV.

Further studies are required to investigate ACE2 expression in other cancers (COAD, KIRP, PAAD, READ, and STAD). Jia $X$ et al. revealed that the expression of ACE2 in cervical squamous cell carcinoma and endometrial adenocarcinoma, kidney renal clear cell carcinoma, KIRP, and PAAD was higher than that in surrounding tissues [25]. The present study not only provided evidence of high ACE2 expression in KIRP and PAAD but also discovered markedly increased levels of ACE2 in COAD, READ, and STAD. Therefore, we suspect that patients with these cancers may be more susceptible to 2019-nCoV, and they are the key protection targets in epidemic prevention work. However, there was no significant difference in the expression of ACE2 among patients aged 21-40 years, 41-60 years, 61-80 years, and 81-100 years. Further large-scale studies are needed to verify these findings. Special attention should be given to cancer patients clinically, noting that they may have a longer course of the illness or a higher risk of severe illness.

Subsequently, the RTK pathway was activated by ACE2. RTKs can be expressed in many cell types, including cells in the tumor microenvironment [26]. As a key regulator of cancer development, the RTK pathway plays an important role in the proliferation, invasion, angiogenesis, and metastasis of cancer [27]. These results suggest that ACE2 plays an important role in tumorigenesis and development. In addition, we found that PI3K/AKT and RAS/MAPK pathways activated by ACE2. Besides, we found that the EMT pathway was inhibited by ACE2, but was activated by AGT, AGTR1, DPP4, MME, PRCP, and XPNPEP2. The deregulation of the cell cycle is a fundamental process that underlies cancer proliferation [28]. We found that some genes were mainly inhibited in the cell cycle, especially ACE2, AGTR1, and DPP4. Although we identified a potential correlation between ACE2 expression and these pathways, whether they are involved in regulating ACE2 expression is worthy of future study.

\section{Conclusion}

Our study aimed to better understand the underlying mechanisms and function of ACE2 with the utilization of extensive databases. Our results demonstrate the importance of ACE2 in LUAD and provide insights into the regulatory mechanisms and functions of ACE2. We hope that these findings provide useful information on the treatment and prevention of 2019-nCoV.

\section{Abbreviations}


ACE2: angiotensin-converting enzyme 2; LUAD: lung adenocarcinoma; CNVs: copy number variations; SNVs: single nucleotide variations; COAD: colon adenocarcinoma; KIRP: kidney renal papillary cell carcinoma; PAAD: pancreatic adenocarcinoma; READ: rectum adenocarcinoma; STAD: stomach adenocarcinoma; COVID-19: 2019 coronavirus disease; WHO: The World Health Organization; SARS-CoV: SARS coronavirus; GO: Gene Ontology; KEGG: Kyoto Encyclopedia of Genes and Genomes; TCGA: The Cancer Genome Atlas; GEPIA: Gene Expression Profiling Interactive Analysis; GTEx: Genotype-Tissue Expression; AGT: Angiotensinogen; REN: Renin; MME: Neprilysin; DPP4: Dipeptidyl peptidase 4; PRCP: Lysosomal Pro-X carboxypeptidase; MEP1A: Meprin A subunit alpha; AGTR1: Type-1 angiotensin II receptor; MEP1B: Meprin A subunit beta; XPNPEP2: Xaa-Pro aminopeptidase 2; AGTR2: Type-2 angiotensin II receptor; NOS: not otherwise

specified; RTK: receptor tyrosine kinase.

\section{Declarations}

\section{Acknowledgements}

We want to thank acknowledge the database available to us for this study.

\section{Authors' contributions}

Q H and $Z \mathrm{~L}$ designed the work and wrote the manuscript. $\mathrm{L}$ S provided patient samples. $Z \mathrm{~L}, \mathrm{~S} \mathrm{C}$ and $\mathrm{J} \mathrm{L}$ performed the statistical analysis, Q H participated in the discussion and language editing. N X reviewed the manuscript. All authors read and approved the final manuscript.

\section{Funding}

This project was supported by the Natural Science Foundation of National (81972003), the Natural Science Foundation of Guangdong (2017A030313668), Sanming Project of Medicine in Shenzhen (SZSM201612031), Shenzhen Municipal Government of China (JCYJ20170817171808368, JCYJ20170818085657917, JCYJ20180507184647104, KQTD20170810160226082).

\section{Availability of data and materials}

The authors are grateful to freely available from WHO.

(https://www.who.int/emergencies/diseases/novel-coronavirus-2019), DriverDBv3 (http://driverdb.tms.cmu.edu.tw/), STRING database (https://string-db.org/), UALCAN (http://ualcan.path.uab.edu/index.html), GEPIA database (http://gepia.cancer-pku.cn/), GSCALite (http://bioinfo.life.hust.edu.cn/web/GSCALite/).

\section{Ethics approval and consent to participate}

This study was approved by the Ethics Committee of Shenzhen Second People's Hospital in accordance with the principles of the Declaration of Helsinki. Informed consent from patients for their medical data to 
be used in the study were obtained.

\section{Consent for publication}

All authors have seen and agreed to publish.

\section{Competing interests}

The authors declare that they have no competing interests.

\section{Author details}

${ }^{1}$ Biobank, Shenzhen Second People' s Hospital, First Affiliated Hospital of Shenzhen University, Shenzhen 518035, China. ${ }^{2}$ Institute of Translational Medicine, Shenzhen Second People's Hospital, First Affiliated Hospital of Shenzhen University, Shenzhen, 518035, China. ${ }^{3}$ Guangzhou Medical University, Guangzhou, 510182, China. ${ }^{4}$ University of South China, Hunan, 421001, China.

\section{References}

1. Li Q, Guan X, Wu P, Wang X, Zhou L, Tong Y, Ren R, Leung KS, Lau EH, Wong JY. Early transmission dynamics in Wuhan, China, of novel coronavirus-infected pneumonia. New England Journal of Medicine. 2020.

2. Huang C, Wang Y, Li X, Ren L, Zhao J, Hu Y, Zhang L, Fan G, Xu J, Gu X. Clinical features of patients infected with 2019 novel coronavirus in Wuhan, China. The lancet. 2020; 395(10223):497-506.

3. Chan JF-W, Yuan S, Kok K-H, To KK-W, Chu H, Yang J, Xing F, Liu J, Yip CC-Y, Poon RW-S. A familial cluster of pneumonia associated with the 2019 novel coronavirus indicating person-to-person transmission: a study of a family cluster. The Lancet. 2020; 395(10223):514-523.

4. Cao Y, Li L, Feng Z, Wan S, Huang P, Sun X, Wen F, Huang X, Ning G, Wang W. Comparative genetic analysis of the novel coronavirus (2019-nCoV/SARS-CoV-2) receptor ACE2 in different populations. Cell discovery. 2020; 6(1):1-4.

5. Zhou P, Yang X-L, Wang X-G, Hu B, Zhang L, Zhang W, Si H-R, Zhu Y, Li B, Huang C-L. A pneumonia outbreak associated with a new coronavirus of probable bat origin. nature. 2020; 579(7798):270-273.

6. Yan R, Zhang Y, Li Y, Xia L, Guo Y, Zhou Q. Structural basis for the recognition of SARS-CoV-2 by fulllength human ACE2. Science. 2020; 367(6485):1444-1448.

7. Zhang H, Penninger JM, Li Y, Zhong N, Slutsky AS. Angiotensin-converting enzyme 2 (ACE2) as a SARS-CoV-2 receptor: molecular mechanisms and potential therapeutic target. Intensive care medicine. 2020; 46(4):586-590.

8. Hofmann H, Geier M, Marzi A, Krumbiegel M, Peipp M, Fey GH, Gramberg T, Pöhlmann S. Susceptibility to SARS coronavirus S protein-driven infection correlates with expression of angiotensin converting enzyme 2 and infection can be blocked by soluble receptor. Biochemical and biophysical research communications. 2004; 319(4):1216-1221. 
9. Zhao Y, Zhao Z, Wang Y, Zhou Y, Ma Y, Zuo W. Single-cell RNA expression profiling of ACE2, the putative receptor of Wuhan 2019-nCov. BioRxiv. 2020.

10. Chen Y, Shan K, Qian W. Asians and other races express similar levels of and share the same genetic polymorphisms of the SARS-CoV-2 cell-entry receptor. 2020.

11. Liu SH, Shen PC, Chen CY, Hsu AN, Cho YC, Lai YL, Chen FH, Li CY, Wang SC, Chen M et al. DriverDBv3: a multi-omics database for cancer driver gene research. Nucleic Acids Res. 2020; 48(D1):D863-D870.

12. Gaudet P, Škunca N, Hu JC, Dessimoz C: Primer on the gene ontology. In: The Gene Ontology Handbook. Humana Press, New York, NY; 2017: 25-37.

13. Kuleshov MV, Jones MR, Rouillard AD, Fernandez NF, Duan Q, Wang Z, Koplev S, Jenkins SL, Jagodnik KM, Lachmann A et al. Enrichr: a comprehensive gene set enrichment analysis web server 2016 update. Nucleic Acids Res. 2016; 44(W1):W90-97.

14. Chandrashekar DS, Bashel B, Balasubramanya SAH, Creighton CJ, Ponce-Rodriguez I, Chakravarthi BV, Varambally S. UALCAN: a portal for facilitating tumor subgroup gene expression and survival analyses. Neoplasia. 2017; 19(8):649-658.

15. Huo Q, Li Z, Cheng L, Yang F, Xie N. SIRT7 Is a Prognostic Biomarker Associated With Immune Infiltration in Luminal Breast Cancer. Frontiers in Oncology. 2020; 10.

16. Zhang Y, Yuan Y, Liang P, Zhang Z, Guo X, Xia L, Zhao Y, Shu XS, Sun S, Ying Y et al. Overexpression of a novel candidate oncogene KIF14 correlates with tumor progression and poor prognosis in prostate cancer. Oncotarget. 2017; 8(28):45459-45469.

17. Tang Z, Li C, Kang B, Gao G, Li C, Zhang Z. GEPIA: a web server for cancer and normal gene expression profiling and interactive analyses. Nucleic acids research. 2017; 45(W1):W98-W102.

18. Akbani R, Ng PK, Werner HM, Shahmoradgoli M, Zhang F, Ju Z, Liu W, Yang JY, Yoshihara K, Li J et al. A pan-cancer proteomic perspective on The Cancer Genome Atlas. Nat Commun. 2014; 5(1):3887.

19. Imai Y, Kuba K, Rao S, Huan Y, Guo F, Guan B, Yang P, Sarao R, Wada T, Leong-Poi H. Angiotensinconverting enzyme 2 protects from severe acute lung failure. Nature. 2005; 436(7047):112-116.

20. Saeed I, Anderson J. Lung cancer: staging, imaging and surgery. Surgery (Oxford). 2005; 23(11):401405.

21. Wrapp D, Wang N, Corbett KS, Goldsmith JA, Hsieh C-L, Abiona O, Graham BS, McLellan JS. Cryo-EM structure of the 2019-nCoV spike in the prefusion conformation. Science. 2020; 367(6483):12601263.

22. Zhang C, Liang Y, Ma M-H, Wu K-Z, Dai D-Q. KRT15, INHBA, MATN3, and AGT are aberrantly methylated and differentially expressed in gastric cancer and associated with prognosis. PathologyResearch and Practice. 2019; 215(5):893-899.

23. Célérier J, Cruz A, Lamandé N, Gasc J-M, Corvol P. Angiotensinogen and its cleaved derivatives inhibit angiogenesis. Hypertension. 2002; 39(2):224-228. 
24. Miners JS, Verbeek MM, Rikkert MO, Kehoe PG, Love S. Immunocapture-based fluorometric assay for the measurement of neprilysin-specific enzyme activity in brain tissue homogenates and cerebrospinal fluid. Journal of neuroscience methods. 2008; 167(2):229-236.

25. Jia X, Yin C, Lu S, Chen Y, Liu Q, Bai J, Lu Y. Two things about COVID-19 might need attention. 2020.

26. Butti R, Das S, Gunasekaran VP, Yadav AS, Kumar D, Kundu GC. Receptor tyrosine kinases (RTKs) in breast cancer: signaling, therapeutic implications and challenges. Molecular cancer. 2018; 17(1):34.

27. Chen G, Weng Q, Fu L, Wang Z, Yu P, Liu Z, Li X, Zhang H, Liang G. Synthesis and biological evaluation of novel oxindole-based RTK inhibitors as anti-cancer agents. Bioorganic \& medicinal chemistry. 2014; 22(24):6953-6960.

28. Hanahan D, Weinberg RA. Hallmarks of cancer: the next generation. cell. 2011; 144(5):646-674.

\section{Figures}

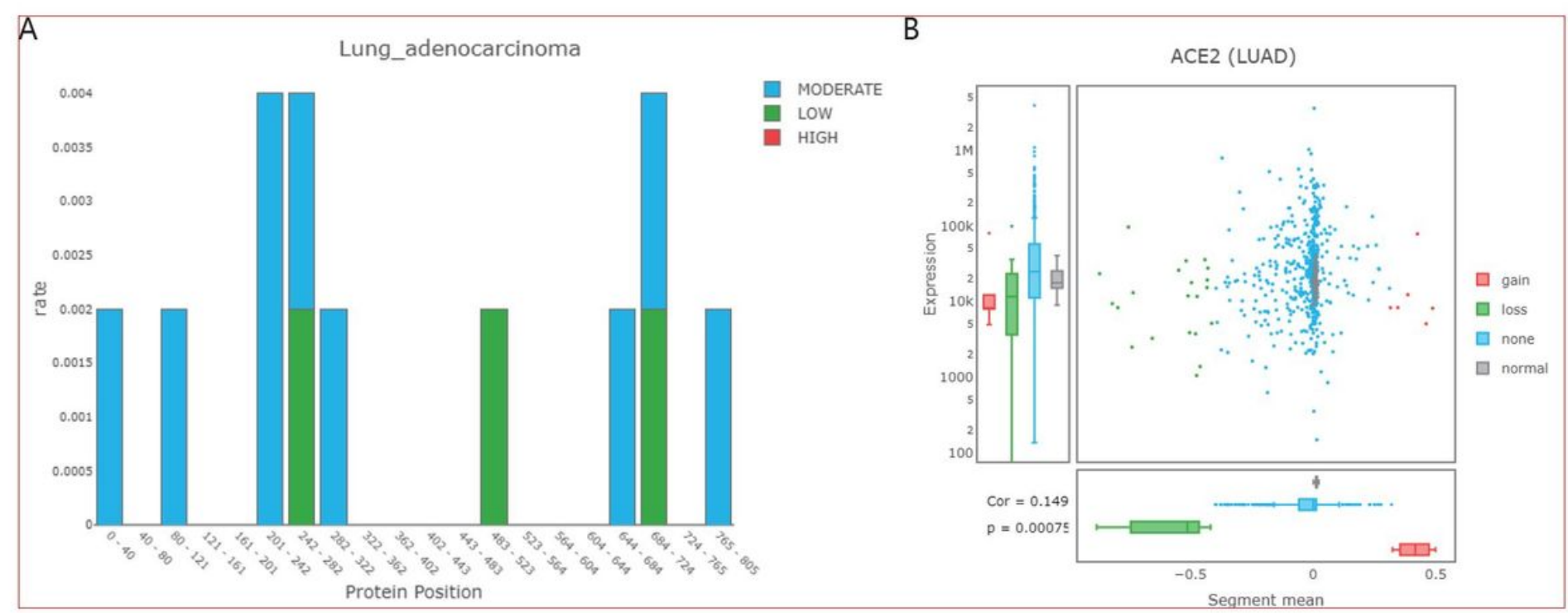

\section{Figure 1}

The mutation rate and CNV distribution of ACE2 analysis in LUAD. A: the mutation rate of ACE2 and its protein positions for LUAD. The mutation squares indicate the number of mutation tools that identify this gene as a mutation driver. B: The CNV squares indicated CNV gain or loss of ACE2 in LUAD. The red represents gain (1) and the green represents a loss (-1). 


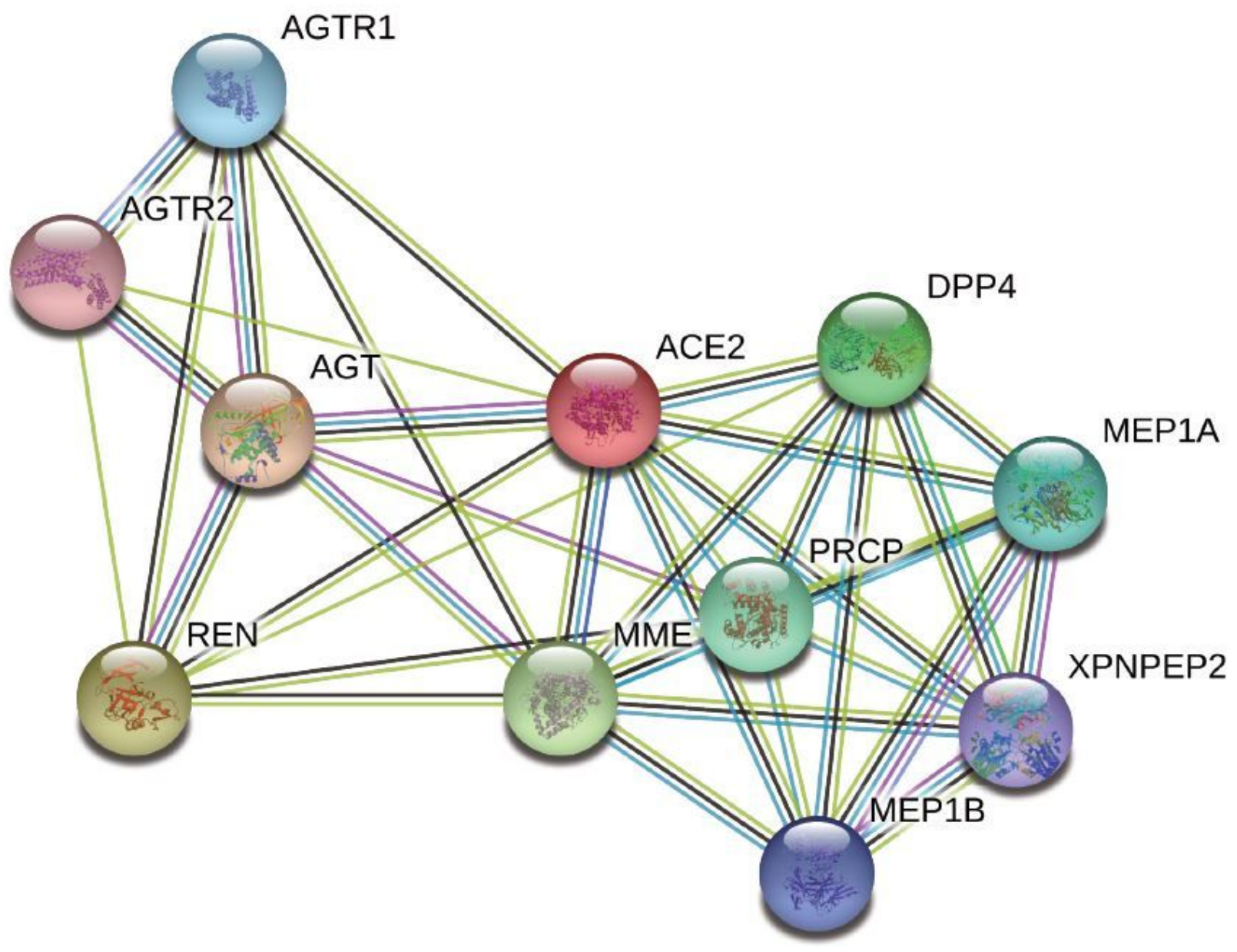

Known Interactions

from curated databases

experimentally determined
Predicted Interactions

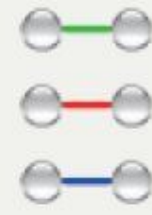

gene neighborhood

gene fusions

gene co-occurrence
Others

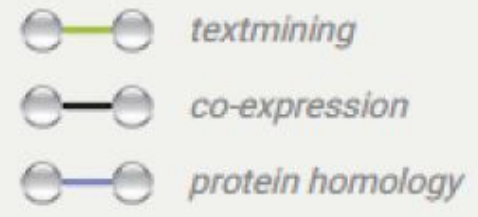

Figure 2

Identification of proteins known and predicted to interact with ACE2 (STRING). 


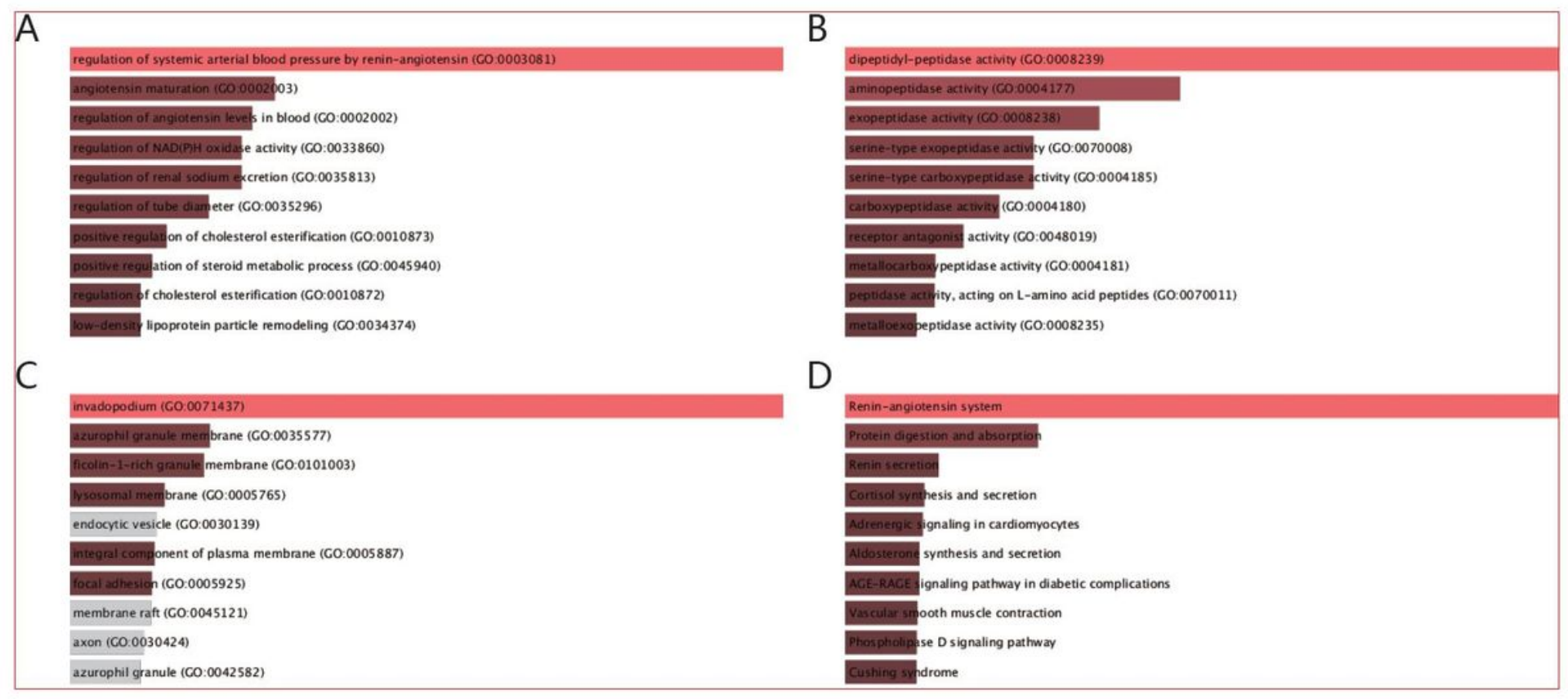

Figure 3

GO enrichment and KEGG pathway analysis via the Enrichr online database. A: biological process; B: molecular function; C: cellular component; D: KEGG pathway analysis.

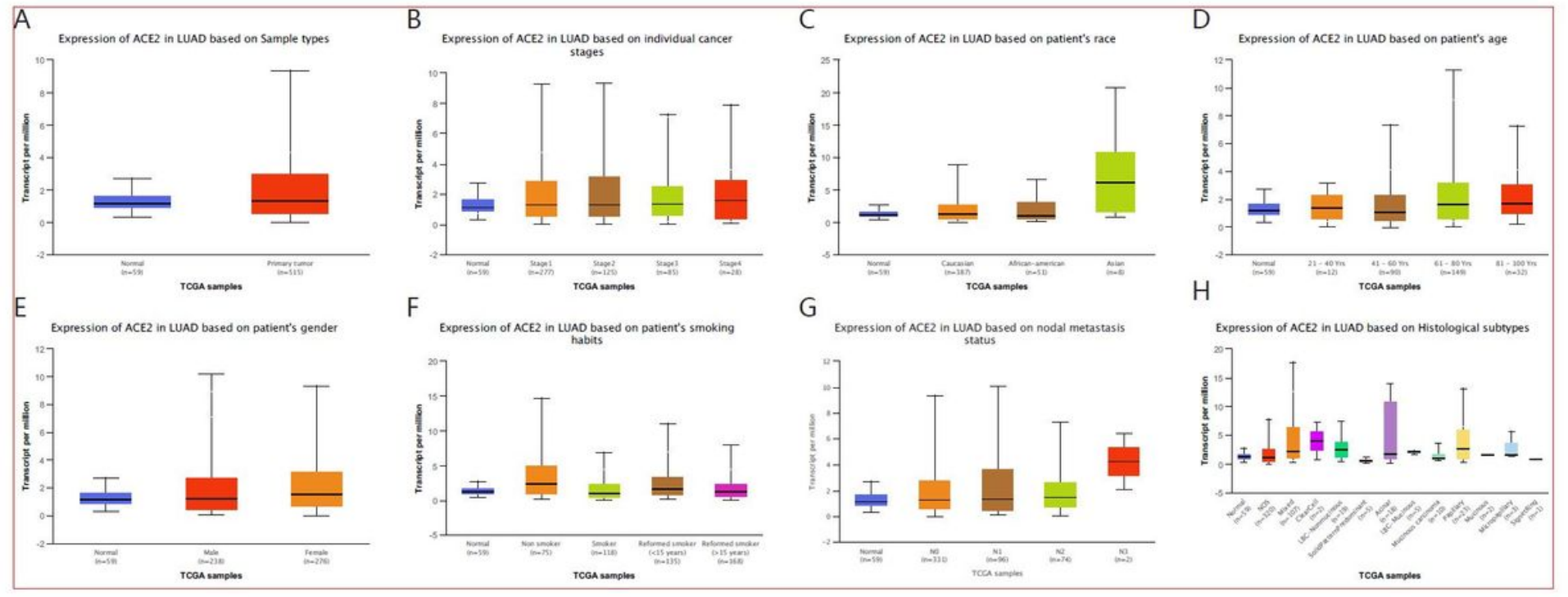

\section{Figure 4}

The correlation between ACE2 mRNA expression level and clinicopathological parameters of breast cancer using UALCAN. A: sample type (normal/primary tumor), B: cancer stage (stages 1, 2, 3, and 4), C: patient's race (Caucasian, African American, and Asian), D: patient's age (21-40; 41-60; 61-80; 81-100), D: patients' gender (Male-vs-Female), E: patient's smoking habits ( Non-smoker, Smoker, Reformed smoker 1 ( $<15$ years), and Reformed smoker 1 (> 15 years), F: node metastasis status (N0: No regional lymph node metastasis; N1: Metastases in 1 to 3 axillary lymph nodes; N2: Metastases in 4 to 9 axillary lymph nodes; 
and N3: Metastases in 10 or more axillary lymph nodes), G: histological subtypes (NOS: Lung Adenocarcinoma-Not Otherwise Specified; Mixed: Lung Adenocarcinoma Mixed subtype; ClearCell: Lung Clear Cell Adenocarcinoma; LBC-Nonmucinous: Lung Bronchioloalveolar Carcinoma Non mucinous; SolidPatternPredominant: Lung Solid Pattern Predominant Adenocarcinoma; Acinar: Lung Acinar Adenocarcinoma; LBC-Mucinous: Lung Bronchioloalveolar Carcinoma Mucinous; Mucinous: ) Mucinous (Colloid) Carcinoma; Papillary: Lung Papillary Adenocarcinoma; Mucinous: Lung Mucinous Adenocarcinoma; Micropapillary: Lung Micropapillary Adenocarcinoma; SignetRing: Lung Signet Ring Adenocarcinoma).

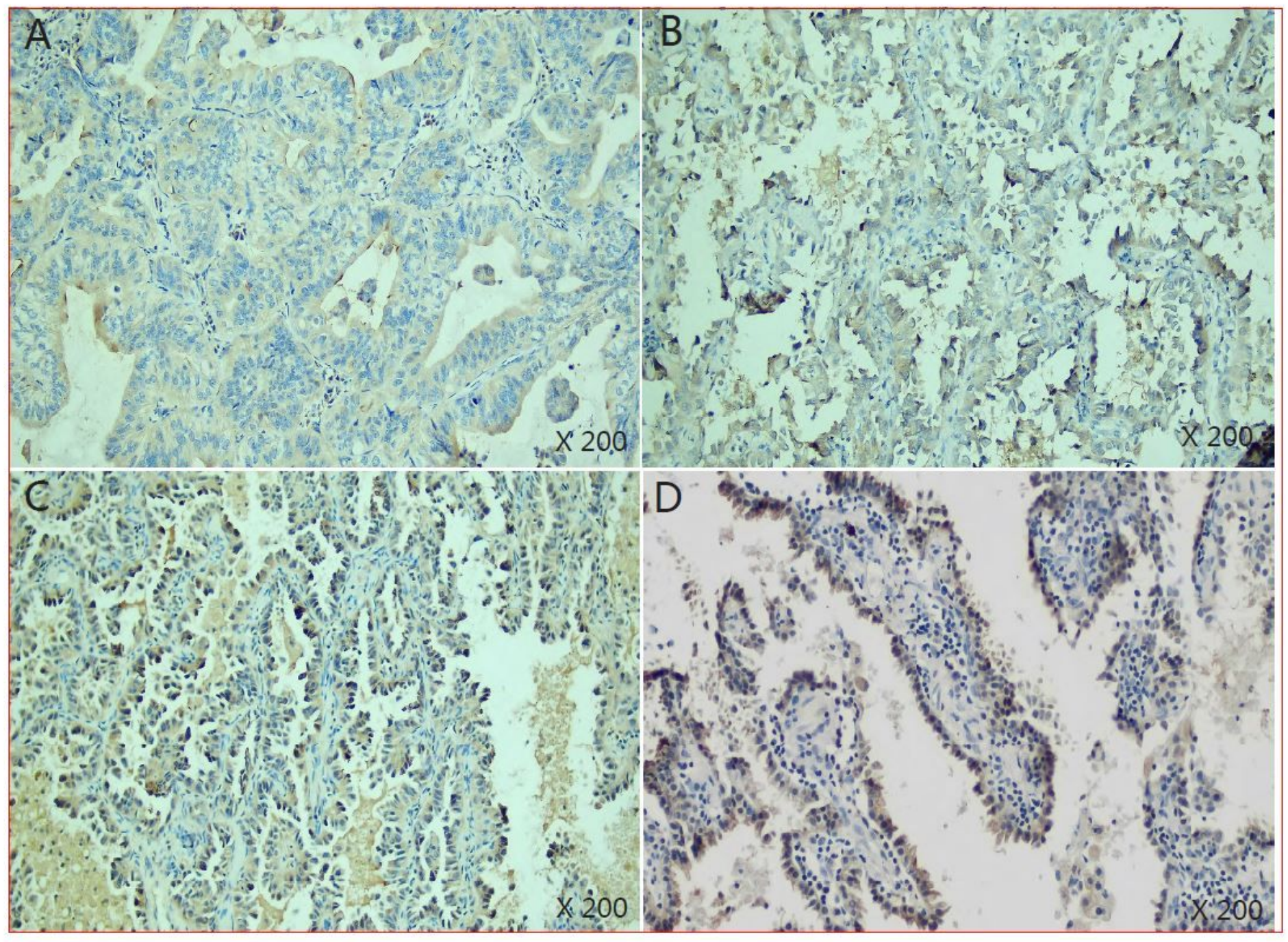

\section{Figure 5}

Levels of ACE2 in patients with LUAD by Immunohistochemical analysis. A: ACE2 expression (-) in patients aged 21-40 years samples. B: ACE2 expression (+) in patients aged 41-60 years samples. C: ACE2 expression (++) in patients aged 61-80 years samples. D: ACE2 expression (+++) in patients aged 81-100 years samples. The expression density of ACE2 in LUAD tissue was quantitated by scoring staining intensity, including negative (-) and weak (+) staining, moderate (++) and strong (+++) staining, respectively. 


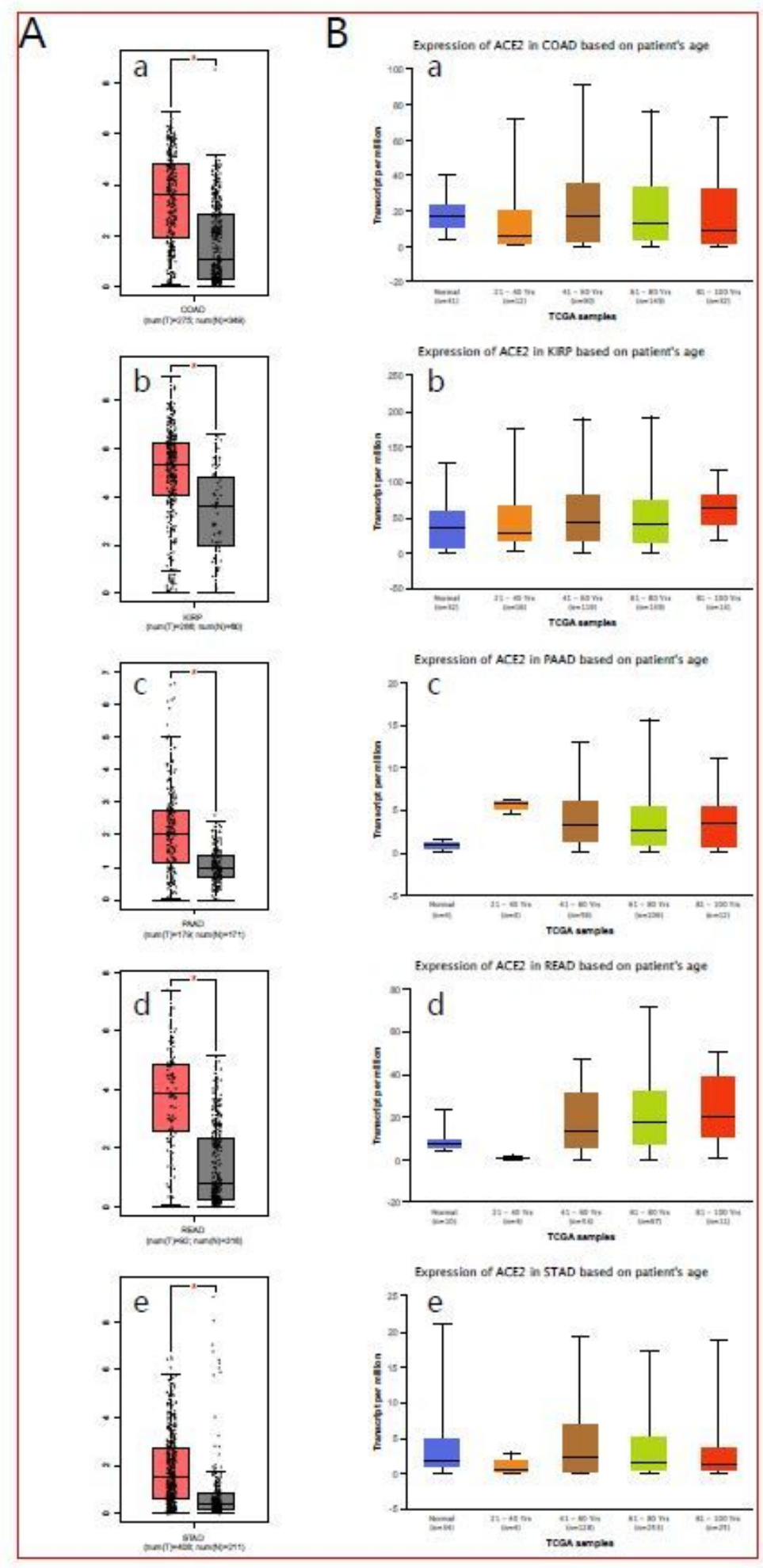

Figure 6

ACE2 expression in other cancers. A: Human ACE2 expression levels in different tumor types from the TCGA database were determined using GEPIA. ACE2 was highly expressed in colon adenocarcinoma (COAD), kidney renal papillary cell carcinoma (KIRP), pancreatic adenocarcinoma (PAAD), rectum adenocarcinoma (READ), and stomach adenocarcinoma (STAD) compared to normal tissues. P-value 
Significant Codes: $0 \leq * \star *<0.001 \leq * *<0.01 \leq *<0.05$. B: The correlation between ACE2 mRNA expression level and patient's age (21-40; 41-60; 61-80; 81-100) in COAD, KIRP, PAAD, READ, and STAD.

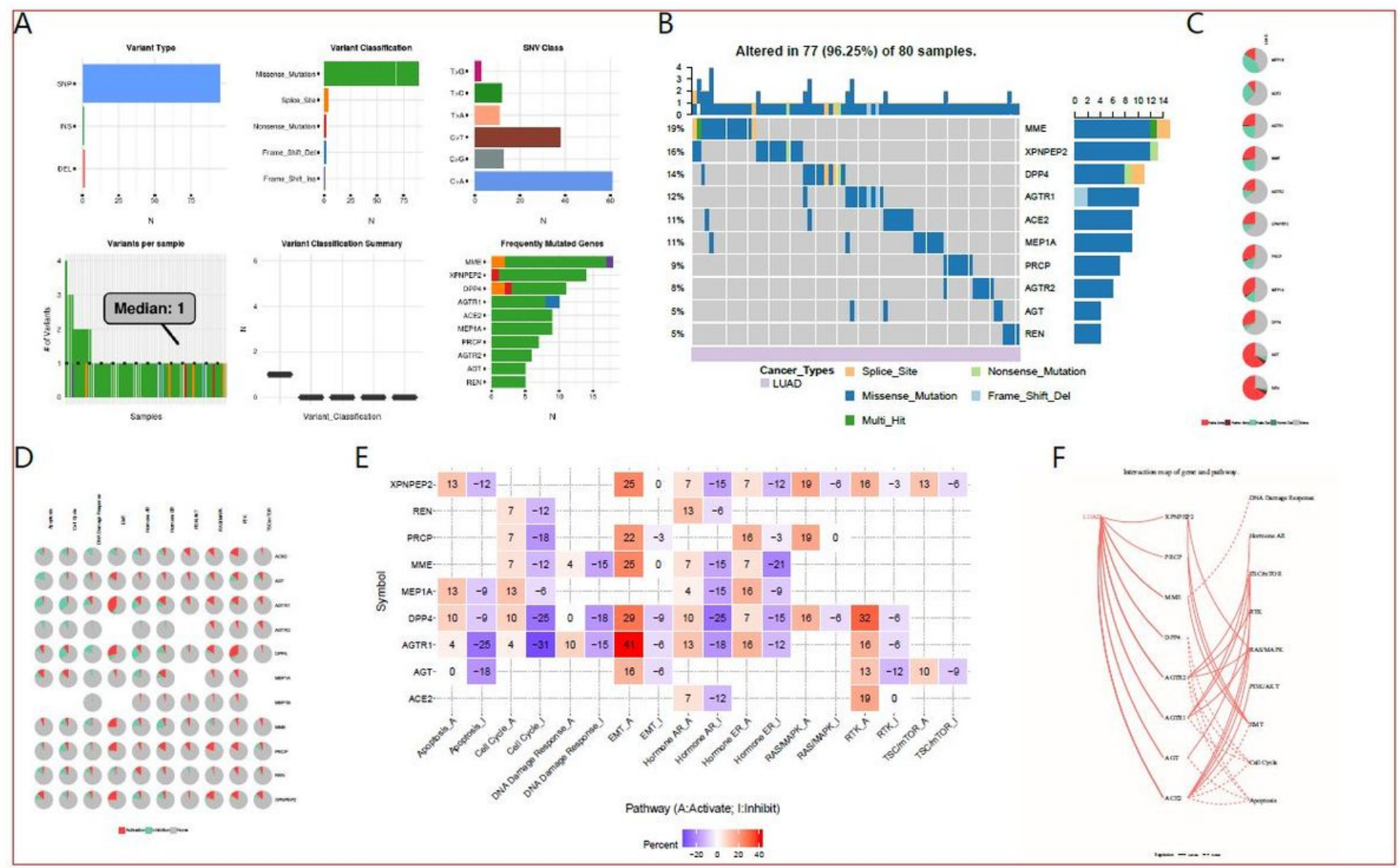

\section{Figure 7}

SNVs, CNVs, and pathway activity analysis in LUAD. A-C: SNVs, CNVs, and pathway activity analysis of the genes interacting with ACE2 by GSCALite. D-F: Pathway activity (proptosis, cell cycle, DNA damage response, EMT, hormone AR, hormone ER, PI3K/AKT, RAS/MAPK, RTK, TSC/mTOR) analysis. 